\title{
Cutaneous actinomycosis. A case report
}

\author{
Tomasz Wasyłyszyn ${ }^{1,2}$, Katarzyna Borowska ${ }^{3,4}$
}

${ }^{1}$ Medical Center Gabinet Dermatologiczny Tomasz Wasylyszyn, 25/421 Generała Andersa Street, 00-159 Warsaw, Poland, ${ }^{2}$ Department of Dermatology, Military Institute of Medicine in Warsaw, Szaserów, 128 Street, 04-141 Warsaw, Poland, ${ }^{3}$ Medical Center CADERM, 87/113 Marszalkowska Street, 00-683 Warsaw, Poland, ${ }^{4}$ Department of Histology and Embryology with Experimental Cytology Unit, Medical University of Lublin, 11 Radziwiłowska Street, 20-080 Lublin, Poland

Corresponding author: Prof. Katarzyna Borowska, E-mail: k_borowska@wp.pl

\begin{abstract}
A 27 year old patient presented a swollen lesion in the right mandibular area. Prior to the visit the patient was diagnosed with acne and was treated for 6 consecutive months with oral limecycline with no positive response. During the visit the cervicofacial actinomycosis was diagnosed and the patient was administered treatment containing oral amoxycilin plus clavulanic acid among others. The skin lesion disappeared within three weeks. The authors discuss this case in spite of diagnostic difficulties of this uncommon condition, especially while differentiating from acne conglobata.
\end{abstract}

Key words: Actinomycosisl; Cicatrical alopecia; Sinuses; Acne conglobata

\section{INTRODUCTION}

The term "Actinomycosis" was derived from the Greek terms aktino, which refers to the radiating appearance of a sulfur granule, and mykos, which labels the condition a mycotic disease. Actinomycosis is a rare subacute or chronic bacterial infection caused by Gram positive, anaerobic or microaerophilic bacilli, Actinomyces spp. These are higher prokaryotic bacteria belonging to the family Actinomyceataceae. Actinomycosis israelii as the causative organism was isolated in 1891 [1].

In most cases the disease spreads endogenously as a secondary infection from the infection foci in the oral cavity, especially from those related to teeth like in the present study. In rare cases though the primary skin actinomycosis may occur [2]. Cervicofacial actinomycosis is the most common form of the disease found in the skin; other forms including soft tissue tumors [3] or coexistence of other bacterial or fungal infections [4] making the diagnosis even more difficult.

\section{CASE REPORT}

A twenty-six years old man presented a skin lesion having the form of reddish indurated complex of nodules, located in the mandibular area (Fig. 1). Patient confirmed the appearance of the lesion from at least one year ago, followed by complicated endodontic treatment. During the next few months the patient was diagnosed with "acne" and treated with systemic limecycline $(300 \mathrm{mg}$ daily for three months, then $150 \mathrm{mg}$ daily for an additional three months) with little or no response.

A physical examination revealed that the lesion was palpably hard, having the consistence of cartilage. It was moderately painful. Submandibular lymph nodes were not swollen. The area over the nodules bore the evidence of scaring with permanent hair loss over the beard (cicatrical alopecia). Further examination showed the presence of the sinuses in the central part of the lesion. Gently pressing the lesion allowed to evacuate a few milliliters of pus which contained small yellowish so called "grains of sulfur".

Considering the above the diagnosis of cervicocranial actinomycosis was stated. The patient was administered orally: amoxycilin plus clavulanic acid $(875 \mathrm{mg}+$ $125 \mathrm{mg}$ ) twice a day for three weeks, nabumetonum $500 \mathrm{mg}$ once a day for ten days; topically an aerosol containing neomycine plus dexamethasone twice a day

\footnotetext{
How to cite this article: Wasyłyszyn T, Borowska K. Cutaneous actinomycosis. A case report. Our Dermatol Online. 2016;7(4):451-452.

Submission: 66.05.2016; Acceptance: 31.08.2016

DOI: 10.7241 /ourd.2016.4.124
}

(c) Our Dermatol Online 4.2016 


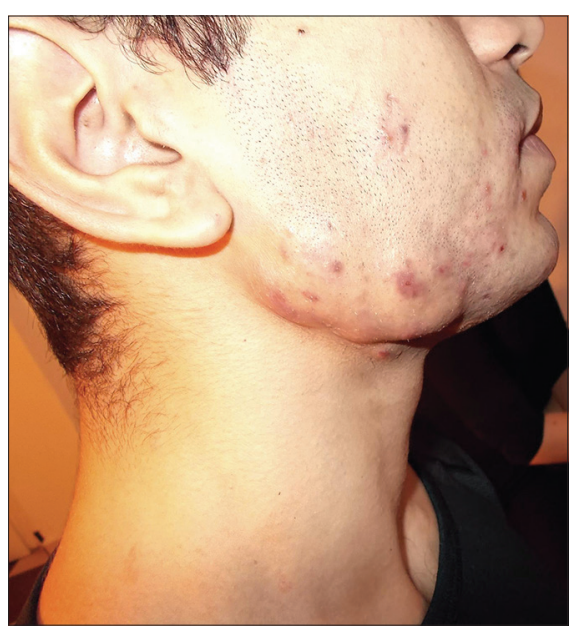

Figure 1: Initial status. Please note swollen area bearing the evidence of cicatrical alopecia.

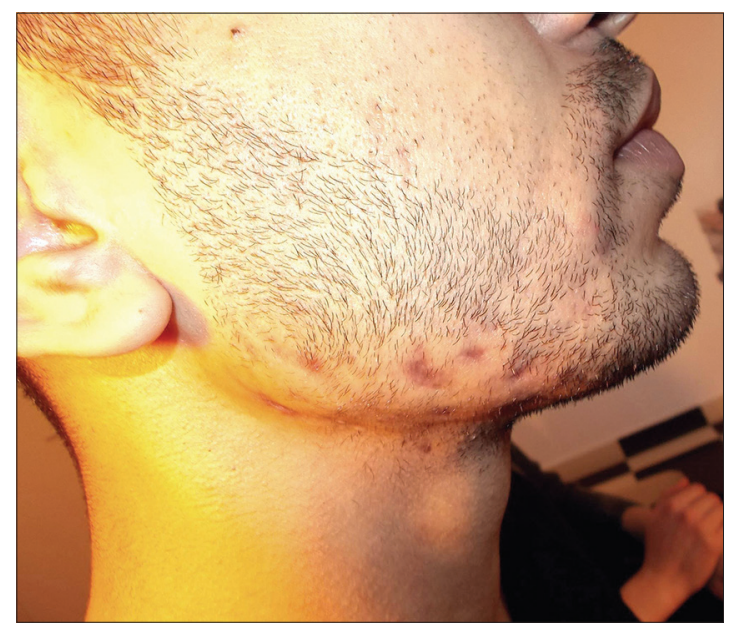

Figure 2: Status after the treatment. The lesion disappeared however the scaring on the skin of the cheek remained.

during whole course of treatment. A control visit after three weeks revealed the disappearance of the lesion however the scaring on the skin of the cheek remained (Fig. 2). The patient was advised to use an ointment containing 2\% chloramphenicol (twice a day for three months) in order to prevent recurrence.

\section{DISCUSSION}

Cervicofacial actinomycosis is typically differentiated from tertiary syphilis and tuberculosis, in the
21 st century though there are very little cases of both, especially in Europe and North America. It seems that younger generations of dermatologists from which the majority have never seen neither tertiary syphilis nor skin tuberculosis may have problems with diagnosing conditions which are clinically similar.

In the present case even given the evidence, well prepared dermatologists may have problems with differentiating actinomycosis from quite common acne conglobata. In both conditions there are nodules, there may be sinuses; the course is long and the location may be similar. In contrary to actinomycosis, acne conglobata lesions are soft, they may be located in upper parts of the face and some lesions disappear while others appear in other places. But the most evident symptom of actinomycosis is the presence of so called "sulfur granules", yellow structures being in fact the colonies of Actinomyces Israeli. The later ones are present in most types of the disease, even in cutaneous fistulas in pulmonary actinomycosis [1].

Unlike acne, actinomycosis responds well to the treatment with penicillins $[1,2]$. In milder cases the antimicrobic treatment accompanied with the administration of anti inflammatory drugs gives satisfactory results. In more serious cases, which may involve the mandibular bone, surgical intervention is necessary.

\section{REFERENCES}

1. Mabeza G.F., Macfarlane J. Pulmonary actinomycosis. Eur Respir J. 2003;21:545-51.

2. Nair PA, Bodiwala NA, Patel SA, Patel KB. A rare case of cutaneous actinomycosis. Indian Dermatol Online J. 2013;4:157-8.

3. Akhtar M, Zade MP, Shahane PL, Bangde AP, Soitkar SM. Scalp actinomycosis presenting as soft tissue tumour: A case report with literature review. Int J Surg Case Rep. 2015;16:99-101.

4. Beniwal NS, Arora S, Baveja S, Sood A, Mehta R, Bal AS. Coexistent Actinomycosis and Eumycetoma in an Immunocompetent Patient. Indian J Dermatol. 2014;59:413-4.

Copyright by Tomasz Wasyłyszyn, et al. This is an open access article distributed under the terms of the Creative Commons Attribution License, which permits unrestricted use, distribution, and reproduction in any medium, provided the original author and source are credited.

Source of Support: Nil, Conflict of Interest: None declared. 